\title{
Jenseits elementarer Formen
}

Das Fazit dieser Untersuchung ist in drei Abschnitte gegliedert: Erstens werden die Grundbegriffe Praxis und Ritualisierung geklärt, zweitens wird die im Verlauf dieser Arbeit verfolgte Argumentation durch ihre Positionierung im Rahmen dreier grundlegender theoretischer Dilemmata rekonstruiert. Schliesslich wird, drittens, eine Antwort auf die Frage danach gegeben, wie jenseits elementarer Formen der Zusammenhang von religiösen Ritualen und sozialer Ordnung beschaffen ist.

\subsection{Praxis und Ritualisierung}

\subsubsection{Praxis}

Praxis wird als eine menschliche Aktivität verstanden, die strukturiert ist und durch ihren Vollzug auf diese Strukturierung zurückwirkt. Mit der Betonung der Strukturiertheit von Handlungen wird darauf hingewiesen, dass sie nicht zufällig oder das Ergebnis situativer Entscheidungen sind, sondern durch die Bedingungen, im Rahmen derer sie stehen und unter denen sie durchgeführt werden, bestimmt werden. Strukturiertheit bedeutet die Erwartbarkeit bestimmter Verhaltensweisen für die Handelnden, für den Wissenschaftler die Erklärbarkeit der Aktivitäten über den Blick auf diese Strukturen. Die Strukturen wiederum werden durch den Blick auf diese Aktivitäten erklärbar, denn diese sind es, die die Strukturen reproduzieren.

Ein solches im Vergleich mit anderen Theorien noch wenig ausgefallen anmutendes Verständnis von Handlung und Struktur gilt es weiter zu spezifizieren. Dazu soll insbesondere identifiziert werden, wie gemäss der vorliegenden Version von Praxistheorie die eben beschriebene Strukturierung des Handelns funktioniert.

Diesbezüglich aufschlussreich ist die Rolle, die darin Wissen und Verbalisierung zugewiesen wird: Die Motivation und das Wissen, das zum Vollzug des Handelns 
notwendig ist, liegt nicht in verbalisierbarer Form vor, sondern ist implizit vorhanden. Die Handelnden folgen einer Regel, die sie nicht explizieren können. Ihnen liegt kein Modell vor, das sie in Handlungen übersetzen. In praxistheoretischen Ansätzen wird die Wirkung von Struktur auf das Handeln nicht in der bewussten Übernahme von und der Entscheidung für bestimmte Handlungsziele gesehen. Und das Handeln funktioniert auch nicht als Befolgung bestimmter gleichermassen bewusst vorhandener Handlungskompetenzen.

Damit ist mehr darüber gesagt, wie die Praxis nicht funktioniert, als wie sie funktioniert: Das Zusammenspiel von Individuum und Gesellschaft erfolgt nicht als Abwägung und Entscheidung auf der Seite des Individuums und der Bereitstellung von geteilten, in einer Logik organisierten Werten auf der Seite der Gesellschaft, die dem Individuum zur Übernahme und Realisierung überlassen werden. Wie, wenn nicht über die Abwägung und Entscheidung unter Massgabe von Werten werden die Selektionen der Individuen angeleitet? Mit dem blossen Verweis auf Unbewusstheit und Implizitheit ist noch nicht erklärt, wie die Strukturierung erfolgt. Sie funktioniert insbesondere über zwei Faktoren:

Erstens sind emotional konstituierte Relevanzen massgeblich. Das Handeln richtet sich an affektiven Präferenzen aus, in Schatzkis Terminologie an einer Teleoaffektivität, die nicht bewusst erkannt oder benannt werden muss. Diese Affektivität dürfte meist wenig enthusiastisch in Form von confidence bestehen, einem unspektakulären Gefühl der Sicherheit, das Richtige auf die richtige Art und Weise zu tun. Zweitens müssen überhaupt die Ressourcen, Fähigkeiten und das Handlungswissen vorhanden sein, die nötig sind, um das affektiv ansprechende Verhalten realisieren zu können. Mit Bourdieu wäre insbesondere auch auf die körperlichen Fähigkeiten hinzuweisen, die praktisch eingeübt werden und für die Reproduktion notwendig sind. Dies weist auf den Charakter der Habitualität hin, der Praxis typischerweise zukommen dürfte: Was bereits eingeübt wurde, wird besser beherrscht und eher mit confidence versehen. Werden Praktiken vollzogen, kann sich damit Teleoaffektivität und Handlungswissen einspielen, was ihre dauerhafte Reproduktion wahrscheinlicher macht. An dieser Stelle wäre mit Bourdieu der Begriff des Habitus anzusiedeln: Damit bezeichnet er kollektive Dispositionen, die Selektionen (z. B. die Präferenz für bestimmte Formen von Kunst bei bestimmten gesellschaftlichen Schichten) wahrscheinlich machen. Diese Wahrscheinlichkeit ist das Ergebnis eines sets von praktisch eingeübten Teleoaffektivitäten und Kompetenzen.

Trotz der Betonung der Implizitheit und Nicht-Bewusstheit stellt Praxis Handeln im Sinne sinnhaften Verhaltens dar. Diejenigen, die eine Praxis vollziehen, sind möglicherweise nicht über die Motive und Bedeutungen informiert, die der Praxis zugeschrieben werden könnten, aber sie können ihr jeweiliges Handeln über das, was David Bloor als glossing nennt, bezeichnen. Eine Person, die eine Kerze anzündet, 
weiss, dass sie gerade eine Kerze anzündet. Dieses glossing ist auch ein framing im Sinne Goffmans, da es eine Antwort auf die Frage, was denn vorgeht, gibt und entsprechende Regeln im oben ausgeführten Sinne mit sich bringt. Damit ist das mit Max Weber gestellte Mindesterfordernis an Handeln als sinnhaftes Verhalten erfüllt - reflexartiges Zwinkern mit den Augen stellt dagegen beispielsweise nur Verhalten dar, nicht Handeln und auch keine Praxis.

Wichtig zur Einordnung dieses Verständnisses von Praxis ist ein grundsätzlicher Unterschied zur Begriffsstrategie Webers. Zunächst einmal steht der Begriff der Praxis, nicht wie bei Weber derjenige des Handelns, im Zentrum, da der Fokus nicht auf einzelnen Akten liegt. Eine noch grundsätzlichere Differenz besteht darin, dass Weber verschiedene Idealtypen des Handelns formuliert. Davon weist einer, das traditionale Handeln, zwar eine gewisse Nähe zu den eben zusammengefassten Betonungen eines Verständnisses von Praxis auf. Praxistheorien formulieren mit „Praxis“ jedoch keinen Idealtyp, sondern einen Universaltyp menschlicher Aktivität. Jegliche Arten des Handelns finden im Rahmen einer Praxis statt. Damit gilt auch ein Handeln, das mit der Explikation von Sinn, mit Reflexivität, einhergeht wie beispielsweise die diskursive Aushandlung von Bedeutungen, als Praxis. Auch auf Diskurse oder Kommunikation trifft das zu, was über Praxis ausgesagt wird, auch sie werden letztlich als durch Strukturen strukturiert gesehen. Die Strukturen können im Rahmen diskursiver Praxis Gegenstand von Reflexivität und Explikation werden, dieser Zugriff ist jedoch einer der Beobachtung, nicht der Konstitution, sie bleiben auch dann Bedingungen des Handelns, die nicht einfach abgeschüttelt werden können. Vielmehr stellt die Reflexion selbst eine Praxis dar, die in bestimmten Strukturen angelegt ist und im Rahmen affektiver Relevanzen und Fähigkeiten umgesetzt wird.

Beispielsweise handelt es sich auch beim Betreiben von Wissenschaft trotz all der darin geforderten Reflexivität um eine teleoaffektiv besetzte, durch implizites Wissen angeleitete Angelegenheit. Der Einzelne in ihrem Rahmen Handelnde kann diese Praxis nicht durch Reflexion und einen darauf basierenden voluntaristischen Kraftakt ohne Weiteres verändern oder aufgeben. Beispielsweise wird das Verfassen einer Habilitationsschrift nicht einfach abgebrochen: Interessen, Fähigkeiten, Karrierechancen, mangelnde Zufriedenheit mit unfertigen Kapiteln, Abgabetermine usw. sind Strukturen, die es wahrscheinlich machen, dass weiter gearbeitet wird. Nicht einmal die praxistheoretisch informierte Bewusstheit über diese quasi im Hintergrund wirkenden Faktoren ändert etwas an ihrer Wirkung.

Die soziologische Diskussion zur Beziehung von Struktur und Handeln beantwortet die hier vorliegende Praxistheorie mit der eingangs genannten Nähe von Praxis zu Struktur und, da Praxis eine Aktivität darstellt, auch zu Handlung. Strukturen stellen, so Niklas Luhmann, aus der Perspektive von Beobachtungen im Feld, 
aber auch seitens des Wissenschaftlers, Erwartungen dar, sind also Konfigurationen der Wirklichkeit, die jeweils bestimmte Anschlüsse nahe legen: Beispielsweise findet sich auf einem Tisch eine Packung Streichhölzer und eine Kerze, die nicht brennt, wobei eine Person an den Tisch heran tritt. Diese Person hegt, wie auch der Beobachter, an diese Ausgangslage bestimmte Erwartungen. Als solche Anschlusswahrscheinlichkeit kommt der Struktur eine virtuelle Dimension zu: Der springende Punkt an der Situation verstanden als Struktur ist nicht das, was passiert (das Wirkliche, das heisst im Moment: Nichts), sondern das, was sie ermöglicht, ja wahrscheinlich macht, nämlich, dass die Person die Kerze anzündet (das Mögliche, das in den Augen der erwartenden Beobachter vor dem Vollzug zur Virtualität wird). Diese virtuelle Struktur kann aufgrund von Erwartungen, damit verbundener Antizipation, Kalkulation oder Unterstellung, auf jeden Fall aber durch mit der Struktur als Erwartung verbundene Teleoaffektivität in der Praxis zu Aktivität und damit realisiert werden. In der Absicht, Licht zu machen, in dem Glauben, einen Auftrag zu befolgen, oder der blossen Freude am Anzünden einer Kerze zündet die Person die Kerze an. Möglicherweise sind Erfahrungen früherer Durchführungen oder explizite Regeln in Form von Anweisungen involviert, es reicht aber auch aus und ist angesichts der Glühbirne, die über dem Tisch glüht und bisher zu erwähnen vergessen wurde, wahrscheinlich -, dass die Person einfach Freude am Handlungsvollzug empfindet, das heisst: Teleoaffektivität und Handlungswissen reichen aus. Im Rahmen von Praxis wird Struktur zum Handeln.

Zweifellos schreiben Praxistheorien dem Menschen, der gewissermassen als Primat der Praxis verstanden wird, eher wenig Souveränität zu. Letztlich ist er jedoch aus einer entsprechenden Sichtweise nicht zum blossen Vollzug übergeordneter Strukturen verurteilt. Strukturen erhöhen nur die Wahrscheinlichkeiten bestimmter Selektionen, sie determinieren sie nicht. Die Realisierung von Strukturen in Handlungen bringt Unbestimmtheiten mit sich, die Improvisationen und Unerwartetes bedingen können. Aus unterschiedlichsten Gründen und Ursachen, Zufällen (Windstoss), Unfähigkeiten (brechendes Streichholz), Launen (Antriebslosigkeit) kann es auch nicht zum Anzünden der Kerze kommen. Zudem ist ein Individuum nicht nur in eine Praxis eingebunden: Es gibt konkurrierende Praktiken mit ihren eigenen Zwängen und so können diese Praktiken gegenseitig den Vollzug irritieren. Man kann, um auf ein etwas lebensnäheres Beispiel zurückzugreifen, nicht den ganzen Tag habilitieren, auch wenn es notwendig oder vielleicht sogar attraktiv scheint. Das Individuum wird dadurch, dass es an mehreren Praktiken zu partizipieren hat, nicht unbedingt freier, aber die einzelne Praxis weniger determinierend.

Das hier vertretene Praxisverständnis bleibt, dies sei als letztes bemerkt, zudem stets eine soziologische Theorie: Wenn Handlungsvollzüge nicht über die eigenmächtigen Selektionen des Individuums verstanden werden, heisst das nicht, dass 
biologische Gegebenheiten, neuronale Muster oder materielle Verteilungen für ihre Erklärung verantwortlich werden. Es wird nach den sozialen Strukturen gefragt, die die Praxis im oben ausgeführten Sinne hervorbringen und die Menschen in diese Aktivitäten einbinden - und die Annahme ist, dass ein Grossteil der beobachteten Varianz in der sozialen Welt darüber verstanden werden kann.

\subsubsection{Ritualisierung}

Ritualisierung ist eine spezifische Art und Weise der Strukturierung von Handlung: Praxis wird nach dem Vorbild früherer Durchführungen vollzogen, sie folgt einer bestimmten Form und Tradition. Ritualisierung kann sich ohne gezielte diesbezügliche Interventionen einspielen, wenn sich beispielsweise regelmässig nach Feierabend die Belegschaft eines Büros zum Umtrunk in einem Gasthaus einfindet. Bei einer solchen stillschweigenden Befolgung von Gewohnheit lässt sich kaum zwischen den drei hier genannten Dimensionen von Ritualisierung, nämlich Formalisierung, Tradition und Repetition, unterscheiden. Ritualisierung kann jedoch auch zu einer von bestimmten Akteuren gezielt angestrebten Unternehmung werden, wodurch die drei Dimensionen auseinander treten: Dann wird von Akteuren im Feld die genaue Repetition früherer Durchführungen einer Praxis gefordert (Repetitivität). Es werden bestimmte Formen, wie beispielsweise spezifische Kleidung, vorgeschrieben, die die jeweilige Praxis von anderen Aktivitäten unterscheidet (Formalisierung), oder die Praxis wird in einer Tradition verortet, die zum Ideal wird (Tradition). Was auf einer Dimension von Ritualisierung versäumt wird, kann auf anderen Dimensionen gewissermassen kompensiert werden. Wenn beispielsweise das beinahe erste Mal ein Papst in den vorzeitigen Ruhestand tritt und das rituell begangen werden soll, kann diesbezüglich nicht auf Repetitivität zurückgegriffen werden. Umso mehr dürfte deshalb bei den Praktiken, die stattfinden, Tradition betont werden, umso wichtiger ist die rituelle Kostümierung und die Beibehaltung anderer Formen wie z. B. der Kommunikation einer feierlichen Stimmung seitens der Beteiligten - so wird klar, dass es sich um ritualisierte Praxis handelt, auch wenn sie so das erste Mal stattfindet.

Wichtig ist die Frage, welche Ebene, diejenige des Gegenstandes oder diejenige des Wissenschaftlers, bei der Definition von Ritualisierung und Ritual welche Rolle spielt. Ritualisierung findet auf der Ebene des Feldes statt, dürfte aber meist erst durch den Wissenschaftler als solche benannt werden. Dabei kann eine ritualisierte Praxis seitens des Wissenschaftlers als ein Ritual bezeichnet werden, wenn auf der Ebene des Untersuchungsgegenstandes bestimmte entsprechende Praktiken über ein glossing gebündelt und als Einheit identifiziert werden - wofür im Untersuchungsge- 
genstand freilich nicht die Kategorisierung als „Ritual“ notwendig ist. Schliesslich ist Ritualisierung eine Frage des Mehr oder Weniger, während Ritual eine dichotome Angelegenheit darstellt. Zu bestimmen, ab welchem Mass von Ritualisierung das Verdikt „Ritual“ angebracht ist, bleibt wiederum dem Wissenschaftler überlassen.

Was sind die Folgen der Ritualisierung von Praxis? Auf einer sehr allgemeinen Ebene mögen dazu Antworten möglich sein. Insbesondere wird die Realisierung von Strukturen, ihr Transfer von Virtualität zu Aktualität, zuverlässig und damit Struktur gestärkt. Die durch die Repetitivität angelegte Wiederholung und das Festhalten an Form und Tradition legt die Häufigkeit sowie die Art und Weise dieser Realisierung zudem fest. Damit sinkt die Flexibilität und Reflexivität wird aus dem Vollzug der Praxis mit zunehmender Ritualisierung ausgeschlossen. Zudem wird mit regelmässiger Durchführung der Ritualvollzug für die Teilnehmenden habitualisiert: Die Kompetenz in der Durchführung, die dafür notwendigen Fähigkeiten und Affektivität spielen sich ein. Wenn das Anzünden der Kerze auf dem Tisch im oben genannten Beispiel ritualisiert ist und die Versuchsperson an dieser Ritualisierung Anteil hatte, ist die Chance gross, dass die Kerze bald brennt.

In den beobachteten Beispielen religiöser Rituale wurden unterschiedliche Folgen von Ritualisierung festgestellt: die zuverlässige Herstellung von Kontakten unter den Teilnehmenden, die Etablierung von Machtverhältnissen, die Plausibilisierung einer Symbolik, oder die Demonstration einer Gruppengrenze. Bevor das weiter ausgeführt wird, gilt es resümierend theoretische Probleme, die im Verlauf dieser mit „Praxis“ und „Ritualisierung“ hantierenden Untersuchung diskutiert werden mussten, zu klären.

\subsection{Theoretische Dilemmata}

Die Arbeit an der Fragestellung dieser Untersuchung mit der hier gewählten praxistheoretischen Position nötigte zur Navigation zwischen verschiedenen theoretischen Dilemmata hindurch. Der entsprechende Kurs wurde unter Rückgriffen auf Mikrosoziologie, Netzwerktheorie und Systemtheorie befolgt, die sich als Ergänzungen zur Praxistheorie wichtig, aber ihrerseits nicht unproblematisch erwiesen.

\subsubsection{Subjektivismus vs. Objektivismus}

Praxistheorien stellen den Versuch dar, die Alternative zwischen Subjektivismus und Objektivismus zu vermeiden. Ersterer würde die soziale Welt über die Entscheidungen des Einzelnen verstehen, letzterer als Realisierung übergreifender Strukturen. 
Dem Subjektivismus wird mit dem praxistheoretischen Handlungsverständnis als erstes abgeschworen. Individuen spielen zwar als Ausgangspunkt von Handlungen, in ihrer körperlichen Anwesenheit und Involviertheit und als Träger von Emotionen eine zentrale Rolle. Aber die Praxis, in der sie diese Rolle spielen, wird, wie eben noch einmal nachgezeichnet, als sozial strukturiert thematisiert. Der Blick auf Rituale rüttelt nicht an diesem Bild: Ritualisierte Praxis kann sich zwar semantisch (evangelikaler Gottesdienst) oder strukturell (Zen-Meditation) auf den Einzelnen beziehen, die Ritualisierung liess sich dennoch als sozial strukturiert entlarven. Auch die beobachteten rituellen Praktiken im Feld alternativer Religiosität, das ohne starke Autoritätsstrukturen auskommt und hohe individuelle Mobilität ermöglicht, reproduziert in hohem Masse homogene Strukturen, die das Individuum nahtlos in die soziale Ordnung der Gesellschaft einfügen.

Auf der Seite des Subjektivismus siedelt Bourdieu auch den Interaktionismus an, den er als Position kritisiert, die die soziale Welt als durch situative Vorgänge strukturiert betrachtet. Die soziale Welt geht nicht aus dem Individuum, sondern aus der Situation hervor. Die Annahme, dass Rituale die über sie hinausgehende soziale Ordnung bestimmen, könnte das nahe legen. Gerade in der Mikrosoziologie, auf die insbesondere in der Version von Randall Collins zur Analyse von Interaktionen zurückgegriffen wurde, findet sich die Auffassung der Situation als Ausgangspunkt des Sozialen. Als praxistheoretisch anschlussfähige Gegenposition konnte auf Erving Goffman Bezug genommen werden, der in Auseinandersetzung mit Mikrosoziologen, die ihn einst als Gewährsmann betrachteten, die Strukturiertheit von Situationen durch frames betonte. Sinnzuschreibungen, Kompetenzen, affektive Strukturen ermöglichen erst die rituellen Situationen, die dann ihrerseits zur Aufrechterhaltung dieser Ordnung beitragen. Den diskutierten Fallbeispielen entspricht das: Die beobachtete ritualisierte Praxis fand nicht als spontane Emergenz statt.

Schwieriger scheint die Vermeidung des Objektivismus. Immer wieder ist in Praxistheorien von Struktur die Rede und oft scheinen Praktiken von übergreifenden Strukturen determinierte Vollzüge darzustellen, die keinen Spielraum für die Situation oder das Individuum offen lassen. Auch die Betrachtung der Fallbeispiele birgt die Gefahr der Überschätzung der strukturierenden Wirkungen von Ritualen. Die Individuen, die sich nicht an die Strapazen der Zen-Meditation gewöhnen, wurden kurz erwähnt, näher betrachtet aber dann doch diejenigen, die sich die notwendigen Fähigkeiten antrainierten. Auch bei den evangelikalen Celebrations standen die Ritualteilnehmer im Fokus, nicht diejenigen, die der Angelegenheit irritiert oder gelangweilt fernblieben. Von Seiten des Forschers kann das idealisierte Zusammenspiel von Struktur und Handlung, das ritualisierte Praxis zu verwirklichen sucht, für 
ein Abbild der weitaus kontingenteren Realität der Gesamtheit der jeweiligen sozialen Ordnung gehalten werden.

Dem wurde hier einerseits systemtheoretisch entgegengewirkt, indem Interaktion als von ihrer gesellschaftlichen Umwelt abgegrenztes System verstanden wurde, das mit spezifischen Begrifflichkeiten zu analysieren ist. Diese Entkopplung verhindert, dass Rituale als Abbild ihres sozialen Kontexts verstanden werden. Wie andere Situationen verfügen sie, mit Goffman gesprochen, über eine Membran, die Einflüsse nicht unvermittelt und notwendigerweise zur Geltung kommen lässt. Nur so ist beispielsweise die Beständigkeit und konservierende Funktion der Todesrituale bei den Zoroastriern in Indien denkbar, die trotz gemeinschaftlichem Aufruhr unirritiert fortgeführt wurden.

Als weitere Massnahme gegen Objektivismus galt es, die sozialen Ordnungen ihrerseits nicht objektivierend oder reifizierend zu konzipieren: So wurden netzwerktheoretisch Gemeinschaften als Gemengelage von Beziehungen, nicht als homogene Einheiten thematisiert. Somit wurde den Gemeinschaften, aber auch der Gesellschaft, nicht mehr die Einheit und Einheitlichkeit zugeschrieben, die Durkheim den Aborigines unterstellte. Diese Einheit wurde nicht als Ausgangspunkt einer in den Worten Bourdieus „Hervorbringung ohne Hervorbringer“ gesehen, sondern als Ziel von Bemühungen und gezielten Ritualisierungen - beispielsweise durch one-to-many-Rituale wie in den Celebrations -, untersucht.

\subsubsection{Idealismus vs. Positivismus}

Das hier verwendete Konzept von Praxis wendet sich gegen die Auffassung, dass Symbole, Werte oder Bedeutungen einer eigenen Sphäre zuzuordnen sind und so etwas wie Symbolsysteme bilden. Es richtet sich auch gegen eine entsprechende Auffassung von Kultur, die die genannten Elemente von einem Gegenüber, das je nachdem als „Gesellschaft“, „Struktur“ oder „soziale Systeme“ bezeichnet wird, unterscheidet. Eine solche Auffassung hat ausgehend von der Durkheiminterpretation von Talcott Parsons, vermittelt über Clifford Geertz, gerade die Religionswissenschaft und ein Bild von „Religion als Kultur“ geprägt.

Ein solches Konzept eines separaten Reichs der Ideen, das einer eigenen Logik folgt, ist praxistheoretisch als idealistisch zu kritisieren. Bedeutungen sind nicht unabhängig von Handlungsvollzügen zu sehen. Diese bestimmen auch die Beziehungen der Symbole untereinander. Eine solche Gegenposition zur Symbolsystemkonzeption kann sich wie Anne Rawls auf das epistemologische Argument der Formes berufen. Eine Herangehensweise über Rituale versucht den Idealismus gezielt dadurch zu vermeiden, dass Strukturen und Handlungen im Zentrum stehen und 
explizite Bedeutungen zwar hinzukommen können, diese aber abgesehen von einer basalen Sinnhaftigkeit nicht notwendigerweise hinzukommen müssen.

Wird so die Autonomie der Kultur verneint, könnte man, so würde Clifford Geertz argumentieren, auf einen Positivismus zusteuern. Diese andere Seite des Dilemmas scheint tatsächlich für Praxistheorien verlockender. Mit einer Position, die Rituale als Resultate übergreifender sozialer Strukturen versteht, handelt man sich Geertz' Vorwurf des „Vulgärpositivismus“ ein. Religion und Ritual würden dabei als blosse Spiegelung gesellschaftlicher Vorgaben gesehen, wie dies beispielsweise dem Marx der Deutschen Ideologie vorgeworfen werden kann. Was als Mittel gegen Objektivismus gilt, könnte auch gegen einen solchen Positivismus helfen: Rituale werden als eigenständige Einheiten gesehen, die kontingent ablaufen und nicht von anderen Einheiten determiniert werden. Das heisst, dass die praxistheoretisch vorgesehene Strukturierung von Ritualen nicht unvermittelt und unausweichlich verläuft, weshalb rituelle Praxis nicht als blosser Reflex einer übergreifenden Ordnung verstanden werden muss. Zudem wirken sie ihrerseits auf diese Strukturen zurück, sind also keine blossen Folgen oder Symptome.

Positivismus im Sinne von Talcott Parsons' Vorwurf der Verkennung der sinnhaften Dimension des sozialen Lebens musste in der vorliegenden Untersuchung ebenfalls problematisiert werden: Gerade die Mikrosoziologie von Randall Collins, dessen Interaktionsrituale zwar Symbole hervorbringen, aber kaum auf ihnen basieren, ist diesbezüglich einseitig. Auch Netzwerktheorien, die für den Blick auf Gemeinschaften fruchtbar gemacht wurden, dürften aufgrund ihrer Konzentration auf formale Merkmale sozialer Beziehungen in den Ruch eines solchen Positivismus geraten. Als Gegenmassnahme war wichtig, mit Durkheim rituelle Praxis nicht als sinnfreie Affektivität oder dumpfe Traditionalität zu sehen, die gerade durch das Ausbleiben von Sinnzuschreibungen gekennzeichnet ist. Eine solche Gegenüberstellung zwischen im Sinne Webers affektuellem oder traditionellem im Gegensatz zu wertrationalem Handeln wurde abgelehnt: Rationalität und Ritualisierung stehen nicht in einem Gegensatz zueinander und dementsprechend sind Rituale nicht als blosses reaktives Verhalten zu sehen.

Praxis ist immer mit einer minimalen Sinnhaftigkeit verknüpft, insofern die Handelnden über den Vorgang des glossing eine Idee davon haben, was sie gerade tun und via frames zumindest entsprechende Regeln aktivieren. Inwiefern Bedeutungen und Modelle dabei verbalisiert und expliziert werden, inwiefern Reflexivität besteht oder nur impliziten Regeln gefolgt wird, wird theoretisch offen gelassen und stellt eine empirische Frage dar. In den diskutierten Fallbeispielen ist der Stellenwert von explizit zugeschriebenen Bedeutungen unterschiedlich: Die Praxis der Kommunen kommt weitgehend ohne komplexe Deutungen aus, und buddhistische Meditation wird gerade so mit frames versehen, dass von Verbalisierungen 
abgesehen wird, während in den evangelikalen Celebrations die Kommunikation theologischer Konzepte eine wichtige Rolle spielt - auf diese Unterschiede wird im letzten Abschnitt zurückzukommen sein. Keiner der Fälle würde jedoch darauf hinweisen, dass Bedeutung und Handeln in verschiedenen Systemen angesiedelt sind.

\subsubsection{Deduktion vs. Geschichte}

Sozialwissenschaft steht seit jeher in der Spannung, eine nomothetische Wissenschaft nach naturwissenschaftlichem Vorbild zu sein und nach Erklärungen zu suchen, die sich in Gesetzen verallgemeinern lassen, oder eine ideographische Wissenschaft nach dem Vorbild der Geschichte zu sein, die ihre Gegenstände in ihrer Spezifität zu verstehen versucht.

Bei Durkheim finden sich nomothetische Tendenzen in Form eines soziologischen Generalisierungsinteresses, einer Verallgemeinerung vom Einfachen auf das Komplexe. In seiner Folge machen im Funktionalismus und Strukturfunktionalismus die Identifikation zeitloser Strukturmerkmale und Funktionskataloge den Kern der Theoriebildung aus. Eine praxistheoretische Position kann auf die eingespielte Kritik an solchen funktionalistischen Generalisierungen aufbauen. Deren deduktivnomothetischer Anspruch hält einer kritischen Überprüfung nicht stand und der Erklärungswert ist nicht so gross, wie beansprucht.

Praxistheorien verfolgen meist keine verallgemeinernde Theoriebildung. In ihren Ursprüngen im Marx der Deutschen Ideologie wendet sich Praxistheorie der Geschichte zu und mit Wittgenstein hat der wichtigste philosophische Pate nicht das Erklären, sondern das Verstehen im Blick, das sich beispielsweise gegen den Evolutionismus von James George Frazer richtete. Andererseits liegt Praxistheorien, auch wenn sie anderweitig keine Universalaussagen anstreben mögen, doch ein universales Verständnis von menschlicher Aktivität zugrunde. Praxis ist ein zeitloser Begriff, ein Verständnis von Struktur und Handlung, das kontextübergreifend angewandt wird. Auch das Verständnis von Ritualisierung verweist auf formale, ahistorische Eigenschaften. Und je wichtiger die mikrosoziologischen Anleihen und der darin enthaltene Interaktionismus in einer Ritualtheorie sind, desto stärker werden Geschichte und Kontext ausgeblendet, und universale Modelle der Struktur von Interaktion halten Einzug.

Darauf wurde, wie im vorherigen Abschnitt gezeigt, reagiert. Jedoch dürften Grundbegrifflichkeiten wie „Handlung“ oder „Praxis“ mit übergreifendem Anspruch schliesslich trotzdem notwendig sein, um überhaupt über verschiedene Kontexte hinweg vergleichen zu können. Entscheidend ist, diese Begrifflichkeiten 
als wissenschaftliche Konzepte zu reflektieren, was gerade der Blick auf die Geschichte dieser Begriffe erlaubt. Dieser Blick führte dazu, dass beispielsweise die Unterstellung der Wichtigkeit von Symbolen in Ritualen, die im Anschluss an Durkheim oft getätigt wird, zurückgenommen wurde. Ob eine explizite Symbolik eine Rolle spielt, wird dem Feld überlassen. Auch die Annahme, dass ritualisierte Praxis von universaler Notwendigkeit für das menschliche Zusammenleben ist, wurde abgelegt. Stattdessen wurde Ritualisierung als Vorgang gesehen, in dem gewisse formale Kriterien der Handlungsstrukturierung wichtig werden können, aber nicht müssen. Die Antwort liefert die Empirie.

Während die Praxistheorie als Referenz solcher Begriffsreflexion dienen konnte, ist sie als Ausgangspunkt der kritischen Auseinandersetzung mit der Historizität von Begriffen weniger geeignet als beispielsweise poststrukturalistische Strömungen, die an der Prominenz des Begriffes ,Diskurs“ zu erkennen sind. Hier wurde zur Historisierung der Konzepte der sozialen Ordnung oder der Religion auf die Systemtheorie Luhmanns zurückgegriffen - diese führt die Geschichtsbezüge fort, die sich beim späteren Talcott Parsons finden, verabschiedet sich jedoch von den ahistorischen Funktionskatalogen, an denen dieser festhielt. Luhmann folgend wurde beispielsweise die Unterscheidung zwischen Interaktion und Gesellschaft als Resultat einer Geschichte der Ausdifferenzierung gesehen, statt auf die formal-soziologische Diskussion der Beziehung zwischen Mikro- und Makroebene des Sozialen zurückzugreifen. Auch „Religion“ wurde als Resultat einer Geschichte der Ausdifferenzierung gesehen und darüber definiert - nicht etwa durch den Verweis auf ein Wesen der Religion oder die analytische Definitionsfreiheit des Wissenschaftlers.

Auf einer solchen Grundlage wurden die Beziehungen real existierender Einheiten untersucht. In der Realität sind sie einem dauerhaften Wandel unterzogen. Das macht die Generalisierung schwieriger, als es Durkheim in seinem Vorhaben vorsah, elementare Formen der Beziehung zwischen Religion und sozialer Ordnung zu identifizieren, die sich auch in komplexeren Verhältnissen wiederfinden liessen.

\subsection{Religiöse Rituale und soziale Ordnung}

Endlich soll ein allgemeines Modell zum Zusammenhang von religiösen Ritualen und sozialer Ordnung präsentiert werden. Es wird von den beobachteten Fällen gestützt und kann mit den beigezogenen Theorien und Konzepten erklärt werden.

Ein zweidimensionales Koordinatensystem stellt die Ausgangslage dafür dar (siehe Abb.6.1). Die X-Achse bildet eine von links nach rechts ansteigende Komplexität ab. Steigende Komplexität bedeutet, dass immer mehr Beziehungen zwischen den Elementen eines Systems möglich sind, wobei nicht alle verwirklicht 


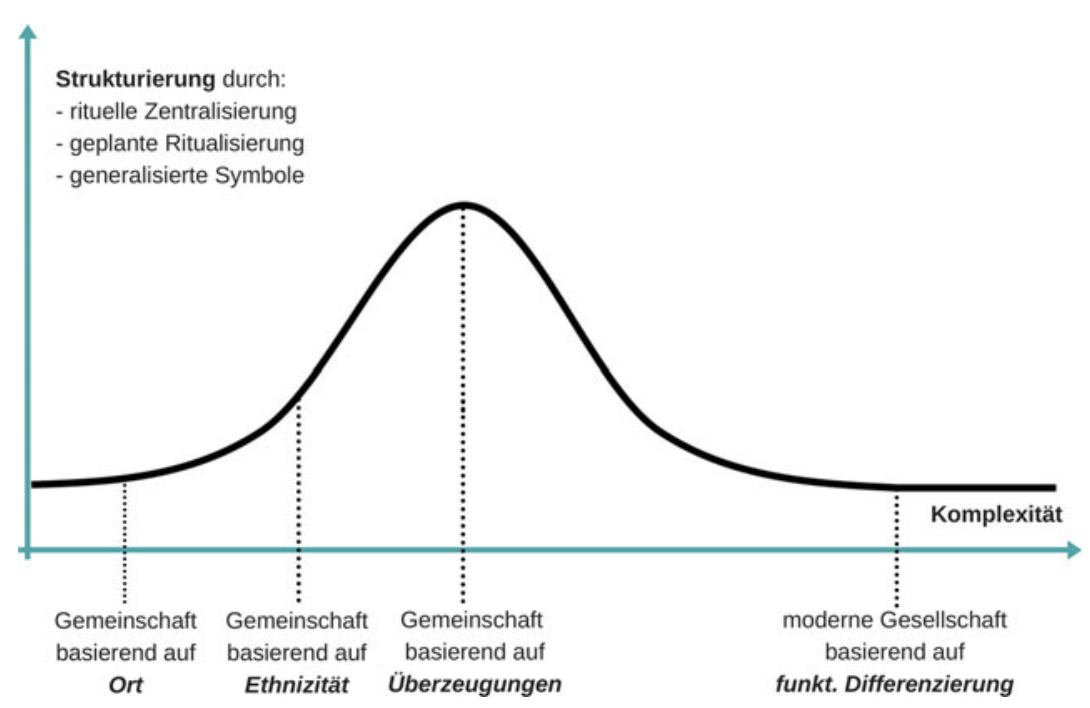

Abb. 6.1 Komplexität und Strukturierung sozialer Ordnungen

werden können. Es geht also nicht um die Kompliziertheit eines Uhrwerks, in dem unzählige Teilchen fest ineinander greifen, sondern um die Merkmale beispielsweise einer Kindergartenklasse mit all ihren Unwägbarkeiten und Entwicklungspotenzialen. Komplexität ist dabei auch nicht als blosse Vervielfältigung elementarer Formen zu verstehen, deren Grundstrukturen im Komplexen multipliziert werden. Komplexität schafft unerwartete und neue Formen, die nicht anhand des Blicks auf einfache Formen vorhergesehen werden können. Eine Interaktion zwischen drei Personen schafft beispielsweise Herausforderungen, die auf der Basis der Kenntnis über Interaktionen zwischen zwei Personen nicht vorhersehbar waren und nicht über diese erklärt oder auf sie zurückgeführt werden können.

Mit der Y-Achse werden gleichzeitig mehrere Faktoren gebündelt, von denen angenommen wird, dass sie stark miteinander korrelieren: Einerseits ist dabei ein Anstieg an Ritualisierung der Praxis gemeint, der gegen oben zudem ein zunehmend absichtlich geplanter Charakter zukommt. Gleichzeitig verschiebt sich gegen oben die Struktur der ritualisierten Praxis immer stärker in Richtung von one-tomany-Kommunikation, und die rituelle Praxis wird immer stärker auf bestimmte Ereignisse hin zentralisiert. Schliesslich findet sich eine abnehmende Konkretheit und zunehmende Abstraktheit der Symbolik, die mit einem stärkeren Generalisierungspotenzial einhergeht. Das heisst, es werden weniger konkrete und lokale Dinge 
symbolisiert (z. B. ein bestimmter Ort), stattdessen finden sich abstrakte Werte (z. B. Liebe), die von sachlichen, zeitlichen oder sozialen Bezügen unabhängig sind.

Das Schema dient nicht dazu, Karrieren einer Gemeinschaft oder religiösen Tradition durch die verschiedenen Sozialformen hindurch darzustellen. Es impliziert auch keine Entwicklungsrichtung oder Ablösung der einen Form durch die andere.

In diesem Schema lassen sich nun die beobachteten Sozialformen - abgesehen von der Interaktion, die hier in ritualisierter Form als Explanans dient - einordnen.

Als Gemeinschaften des Ortes sind Kommunen durch geringe Komplexität geprägt, die sich darin zeigt, dass die Mitglieder in engem Kontakt miteinander stehen und ihre Leben im Rahmen der begrenzten Möglichkeiten der Gemeinschaft führen. Diese gemeinsame Lebensführung besteht in der Praxis der alltäglichen Verrichtungen. Um Kontakte herzustellen, sind kollektive Rituale nicht notwendig, Zeit und Energie dafür sind neben den für die Selbstversorgung aufzubringenden Anstrengungen sowieso knapp. Somit müssen keine rituellen Zusammenkünfte geplant und formalisiert werden. Wo von Ritualisierung überhaupt die Rede sein kann, spielt sie sich wie das Singen während der Arbeit entlang pragmatischer Notwendigkeiten ein. Oder sie widmet sich über kollektive Bekenntnisrituale der Lösung von Problemen, die sich aus dem engen Zusammenleben ergeben. Auch eine generalisierte Symbolik ist nicht notwendig: Die Gemeinschaft ist für alle Beteiligten offensichtlich und eindeutig vom Rest der Welt unterscheidbar, um das zu symbolisieren ist kaum Abstraktion notwendig. Damit kommen Gemeinschaften des Ortes auf der X- wie der Y-Achse niedrige Werte zu.

Eine Gemeinschaft, die sich über ethnische Kriterien definiert, löst sich von der Festlegung auf einen lokalen Lebenszusammenhang, was die Garantie regelmässiger Kontakte zwischen den Gemeinschaftsmitgliedern aufhebt. Der Alltag führt die Mitglieder damit zwar nicht automatisch zusammen, das Leben im ethnisch definierten Zusammenhang bringt jedoch gewisse geteilte Praktiken mit sich, wie z. B. Kleidung oder Heiratsregeln. Kommt wie beim beobachteten Beispiel der Parsi Zoroastrier eine religiöse Grenzziehung hinzu, wird sie durch die ethnisch bestimmte Lebensführung gestützt. Über entsprechende Kriterien ist das Individuum primordial an die Gemeinschaft und damit auch an die Religion gebunden. Seine Teilnahme oder Emotionalität stellt deshalb nicht den primären Bezugspunkt der religiösen Rituale dar, in diesen geht es vielmehr um die Aufrechterhaltung und sichtbare Realisierung der Gemeinschaftsgrenzen, z. B. über den Ausschluss von Nicht-Mitgliedern. Die Reproduktion der Rituale ist durch Traditionalität angeleitet, die Ritualisierung funktioniert als Reproduktion früherer Durchführungen und kann auf Dispositionen und Infrastrukturen zählen, die genau diese (und keine andere) Reproduktion gewährleisten. Die Ritualisierung ist damit gegeben, gezielte Planung ist bloss zu ihrer Sicherstellung notwendig. Wer eine Machtposition 
innerhalb der Gemeinschaft erlangen möchte, muss sich an diesem rituellen Komplex orientieren.

Die in die rituelle Praxis involvierte Symbolik, bei den Parsen die Unterscheidung Reinheit/Unreinheit, setzt an der gemeinschaftlichen Grenzziehung an. Damit ist sie an die rituellen und alltäglichen gemeinschaftlichen Vollzüge gebunden. Durch die Zuordnung von Handlungen, Wesenheiten und Gegenständen auf die Seite der Reinheit oder der Unreinheit gewinnt sie eine alltägliche Konkretion, ist also wenig generalisiert. Immerhin ist das Mass an Ritualisierung, Zentralisierung und Generalisierung der Symbolik im Vergleich zu den Gemeinschaften des Ortes grösser.

Eine auf Überzeugungen basierende Gemeinschaft, in Tönnies' Worten eine Gemeinschaft des Geistes, basiert auf der Übernahme von Überzeugungen seitens ihrer Mitglieder, beispielsweise von religiösen Glaubensvorstellungen. Dabei kann sie sich nicht auf örtliche oder ethnische Gemeinsamkeiten verlassen. Damit sind die Bindungen der Individuen nicht durch den Alltag oder primordiale Merkmale gegeben, was die Mobilität der Individuen und damit auch die Komplexität der Gemeinschaft erhöht. In einer solchen Gemeinschaft, die sonst nicht in praktischen Vollzügen versammelt wäre, sind Rituale für das Schaffen von Kontakten entscheidend. Sie integrieren die Individuen in Beziehungen zu anderen Mitgliedern und sie kommunizieren und plausibilisieren auf emotionale Art und Weise die geteilten Glaubensvorstellungen. Diese decken im Vergleich zu den anderen Gemeinschaftsformen eine komplexere soziale Ordnung ab, damit ist auch die Symbolik komplexer, kann nicht an einem konkreten Ort und nicht einmal einer festen Gruppe festgemacht werden, sondern muss die Mitglieder in ihrem Voluntarismus der Zugehörigkeit und der Mobilität ihrer Lebensführung, die sie immer wieder aus der Gemeinschaft hinaus führen, abdecken. Deshalb muss eine systematisierte und generalisierte Symbolik entwickelt werden und entsprechend wird auch deren Ritualisierung zum Produkt zielstrebiger Bemühungen. Eine generalisierte Symbolik muss wiederum ritualisierbare Konkretion erhalten. Die hohe Komplexität der Ausgangslage auf der X-Achse, so lässt sich dies zusammenfassen, geht mit hoher ritueller Zentralisierung, one-to-many-Ritualen und hochgradig generalisierter Symbolik einher. Die Explizitheit der Symbolik und die Ausdifferenziertheit der Rituale machen es einfach, in dieser Form von Gemeinschaft Religion zu erkennen. Entsprechend ist sie der Ausgangspunkt für Religionsdefinitionen und der zweifelhafte Prototyp für daran ansetzende Theoriebildung.

Noch weiter steigt die Komplexität, wenn der Blick auf die soziale Ordnung der modernen Gesellschaft fällt. Die funktionale Differenzierung stellt einen Gegensatz zur Form der Gemeinschaft dar. Vergesellschaftete Beziehungen sind sachlich spezifisch und zeitlich begrenzt, was in einem dadurch geprägten sozialen Ganzen zu 
einer höheren Dynamik, einer grösseren Anzahl uneingelöster Anschlussmöglichkeiten und damit Unvorhersehbarkeiten führt.

Eine Überführung einer solchen Ordnung in einheitsstiftende one-to-manyRituale ist praktisch nicht mehr möglich. Zwar können Grossrituale massenmedial vermittelt werden, doch die Medien sind ihrerseits komplex, stehen in Konkurrenz zueinander, filtern und selegieren die übermittelten Inhalte und werden ihrerseits selektiv konsumiert oder gar nicht erst empfangen. Eine Symbolik, die angesichts der Komplexität der Gesellschaft und der Individualität ihrer Mitglieder konsensfähig wäre, müsste in so hohem Masse generalisiert sein, dass sie jegliche Verbindlichkeit verliert. Im Rahmen von Zivilreligion wird all dies dennoch versucht, doch auch wenn dies gelingen würde, wären dadurch hergestellte Einheiten bloss Nationen und damit Gemeinschaften, die sich durch je spezifische Konfigurationen von Ethnizität, Ort und Geist definieren. Die Gesellschaft lässt sich so nicht integrieren.

Auch wenn religiöse Rituale zur Konstitution und Reproduktion von Gemeinschaften beitragen, tun sie das innerhalb der Gesellschaft, denn die Gemeinschaften sind schliesslich Teil von ihr. Die Gesellschaft selbst ist jedoch keine Gemeinschaft mehr, sie kann sich nicht mehr versammeln und konzentrieren, wie es Durkheim vorschwebte. Religiöse Rituale, die sich auf die soziale Ordnung der Gesellschaft beziehen und damit am ehesten die Nachfolge von Durkheims Kollektivritualen antreten, passen sich der Komplexität der Gesellschaft an. Sie bedürfen keines bestimmten Personenkreises und keiner übergreifender Institutionen, ihre Referenz ist nicht die Gemeinschaft, sondern das Individuum. Ihr Vollzug und ihre Wirkungen sind dezentral, schwieriger zu identifizieren und hinsichtlich ihrer Relevanz schwieriger zu beurteilen als die kollektiven Rituale der Aborigines oder die Grossrituale der Zivilreligion.

In der Einleitung der Formes beschreibt Durkheim die Vielfalt der Gesellschaft und der Formen religiöser Rituale darin mit dem Bild einer verschwenderischen Vegetation, einer „luxuriante végétation“. Der darunter liegende „,fond commun“ des religiösen Lebens, aus dem die Vielfalt spriesse, sei kaum mehr zu erkennen. Aus dem, was Durkheim von den seiner Meinung nach elementaren Formen von Religion lernte, zog er den Schluss, dass diese den zentralen Faktor bei der Erzeugung von Solidarität und der Aufrechterhaltung sozialer Ordnung darstellen. Wie das in der modernen Gesellschaft genau ablaufen soll, konnte er dabei jedoch nicht überzeugend darlegen.

100 Jahre später ist das Dickicht nicht lichter geworden und mit einem Waldboden rechnet man nicht einmal mehr. Denn dem Gegenstand Religion wäre der Versuch, ihn auf ein festes Substrat zurückzuführen, nicht angemessen. Die Möglichkeit, anhand elementarer Formen auf komplexere Formen zu schliessen, ist deshalb auszuschliessen, was die Ausgangslage für die Theoriebildung erschwert. Ebenfalls 
unmöglich ist es, soziale Ordnungen durch das Eruieren eines zentralen und universalen Faktors zu erklären. Die Antwort auf die Frage der Wirkung von Religion auf das Soziale kann somit auch keine einfache sein. Die Wissenschaft, das wusste bereits Durkheim, lehrt als erstes, wie komplex alles ist. Die in diesem Buch vorgenommene Analyse ist ein Beitrag dazu, einen Teil dieser Komplexität zu verstehen, ohne im Gestrüpp hängen zu bleiben.

Open Access Dieses Kapitel wird unter der Creative Commons Namensnennung 4.0 International Lizenz (http://creativecommons.org/licenses/by/4.0/deed.de) veröffentlicht, welche die Nutzung, Vervielfältigung, Bearbeitung, Verbreitung und Wiedergabe in jeglichem Medium und Format erlaubt, sofern Sie den/die ursprünglichen Autor(en) und die Quelle ordnungsgemäß nennen, einen Link zur Creative Commons Lizenz beifügen und angeben, ob Änderungen vorgenommen wurden.

Die in diesem Kapitel enthaltenen Bilder und sonstiges Drittmaterial unterliegen ebenfalls der genannten Creative Commons Lizenz, sofern sich aus der Abbildungslegende nichts anderes ergibt. Sofern das betreffende Material nicht unter der genannten Creative Commons Lizenz steht und die betreffende Handlung nicht nach gesetzlichen Vorschriften erlaubt ist, ist für die oben aufgeführten Weiterverwendungen des Materials die Einwilligung des jeweiligen Rechteinhabers einzuholen. 